\title{
RECTANGULAR ISOTROPIC KIRCHHOFF-LOVE PLATE ON AN ELASTIC FOUNDATION UNDER THE ACTION OF UNSTEADY ELASTIC DIFFUSION PERTURBATIONS
}

\author{
ZEMSKOV V. ANDREI ${ }^{1,2}$, TARLAKOVSKII V. DMITRY ${ }^{2,1}$ \\ ${ }^{1}$ Moscow Aviation Institute (National Research Institute) \\ 125993, Moscow, Volokolamskoe shosse, 4, Russia \\ azemskov1975@mail.ru \\ 2 Research Institute of Mechanics, Lomonosov Moscow State University \\ 119192, Moscow, Michurinsky prospekt 1, Russia \\ tdvhome@mail.ru
}

Key words: elastic diffusion, Laplace transform, Fourier series, Green's function, Kirchhoff-Love plate, elastic foundation, unsteady problem

\begin{abstract}
We study unsteady elastic diffusion vibrations of a freely supported rectangular isotropic Kirchhoff-Love plate on an elastic foundation, which is under the action of a distributed transverse load. A model that describes coupled elastic diffusion processes in multicomponent continuum is used for the mathematical problem formulation. The longitudinal and transverse vibrations equations of a rectangular isotropic Kirchhoff-Love plate with diffusion were obtained from the model using the d'Alembert variational principle.
\end{abstract}

The problem solution of unsteady elastic diffusion plate vibrations is sought in integral form. The bulk Green's functions are the kernels of the integral representations. To find the Green's functions, we used the Laplace transform in time and the expansion into double trigonometric Fourier series in spatial coordinates. Green's functions in the image domain are represented in the form of rational functions depends on the Laplace transform parameter. The transition to the original domain is done analytically through residues and tables of operational calculus. The bulk Green's functions analytical expressions are obtained.

Using a two-component continuum, a numerical study of unsteady mechanical and diffusion fields interaction is done for an isotropic plate. The solution is presented in analytical form, as well as in the form of three-dimensional graphs of the displacement fields and concentration increments on time and coordinates.

\section{INTRODUCTION}

A review of modern publications shows that the issues related to the study of the interaction of different physical nature fields is relevant. In particular, the load-carrying capacity of individual structural elements such as beams, plates and shells can be influenced by diffusion processes arising from mechanical loads. These issues are discussed in the articles [1,2], where the influence of diffusion processes on the bearing capacity of a shallow transversally isotropic shell is investigated. The publications $[3,4,5]$ 


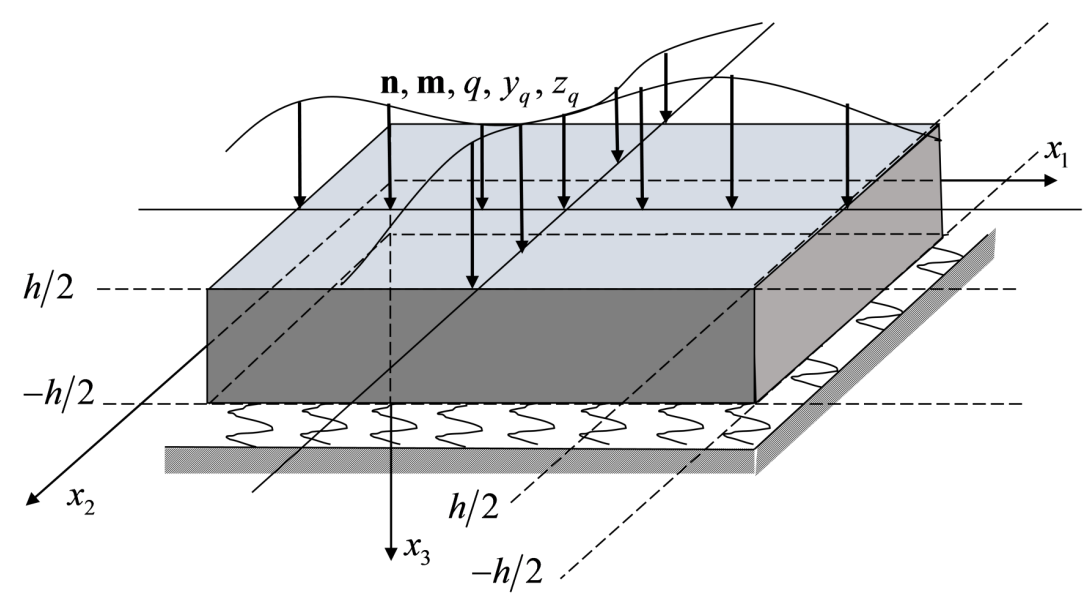

Figure 1: Forces and moments acting upon the plate

are devoted to the study of mechanodiffusion processes in plates. The calculation of an elastic spherical shells with diffusion is considered in [6].

It should be noted that all these problems are solved in a stationary formulation. The problems statements of unsteady elastic diffusion vibrations of beams and plates and methods for their solution are absent in the publications known to date.

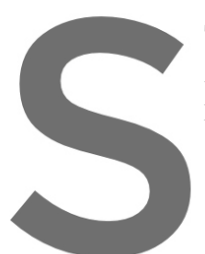

This article considers the plate on an elastic founda

based on variational principles a

$[7,8,9,10]$.

2 GENERAL ESPECTHICATTONS
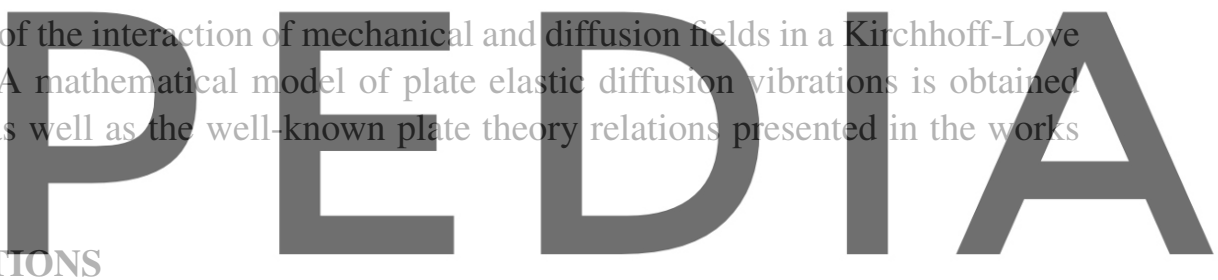

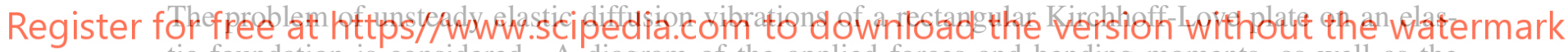
tic foundation is considered. A diagram of the applied forces and bending moments, as well as the orientation of the axes of a rectangular Cartesian coordinate system is shown in the figure 1 .

Here $n=\left\{n_{1}, n_{2}\right\}$ is a longitudinal load density; $m=\left\{m_{1}, m_{2}\right\}$ is a moment density (bending moment per unit surface); $q$ is a transverse load density; $y_{q}, z_{q}$ are linear distribution of diffusion volume source.

For the problem formulation, we use the coupled $N$-component elastic diffusion continuum model in a rectangular Cartesian coordinate system, which has the next form $[11,12,13]$ :

$$
\ddot{u}_{i}=\frac{\partial \sigma_{i j}}{\partial x_{j}}+F_{i}, \dot{\eta}^{(q)}=-\frac{\partial J_{i}^{(q)}}{\partial x_{i}}+Y^{(q)}, \eta^{(N+1)}=-\sum_{q=1}^{N} \eta^{(q)}(q=\overline{1, N}) .
$$

where $\sigma_{i j}$ and $J_{i}^{(q)}$ are the stress tensor and the diffusion flux vector components respectively, which are defined as follows $($ anindexq $=\overline{1, N})$ :

$$
\sigma_{i j}=C_{i j k l} \frac{\partial u_{k}}{\partial x_{l}}-\sum_{q=1}^{N} \alpha_{i j}^{(q)} \eta^{(q)}, J_{i}^{(q)}+\tau_{q} j_{i}^{(q)}=-\sum_{t=1}^{N} D_{i j}^{(q)} g^{(q t)} \frac{\partial \eta^{(t)}}{\partial x_{j}}+\Lambda_{i j k l}^{(q)} \frac{\partial^{2} u_{k}}{\partial x_{j} \partial x_{l}} .
$$


Here, the dots denote are time derivatives. All quantities in (1) and (2) are dimensionless. For them the following notation is used

$$
\begin{gathered}
x_{i}=\frac{x_{i}^{*}}{l}, u_{i}=\frac{u_{i}^{*}}{l}, \tau=\frac{c t}{l}, C_{i j k l}=\frac{C_{i j k l}^{*}}{C_{1111}^{*}}, C^{2}=\frac{C_{1111}^{*}}{\rho}, \alpha_{i j}^{(q)}=\frac{\alpha_{i j}^{*(q)}}{C_{1111}^{*}}, l_{m}=\frac{l_{m}^{*}}{l}, \\
D_{i j}^{(q)}=\frac{D_{i j}^{*(q)}}{C l}, \Lambda_{i j k l}^{(q)}=\frac{m^{(q)} D_{i j}^{*(q)} \alpha_{k l}^{*(q)} n_{0}^{(q)}}{\rho R T_{0} C l}, F_{i}=\frac{\rho l F_{i}^{*}}{C_{1111}^{*}}, Y^{(q)}=\frac{l Y^{*(q)}}{C}, h=\frac{h^{*}}{l}, \tau_{q}=\frac{c \tau^{(q)}}{l},
\end{gathered}
$$

where $t$ is time; $x_{i}^{*}$ are the rectangular Cartesian coordinates; $u_{i}^{*}$ are the displacement vector components; $l$ is the characteristic linear size of the problem; $l_{1}^{*}, l_{2}^{*}$ are the dimensions of the plate; $h^{*}$ is the thickness of the plate; $\eta^{(q)}=n^{(q)}-n_{0}^{(q)}$ is the concentration increment of $q$-th component in the multicomponent continuum; $n^{(q)}$ and $n_{0}^{(q)}$ are the actual and initial concentrations; $C_{i j k l}^{*}$ are elastic constant tensor components; $\rho$ is the medium density; $\alpha_{i j}^{*(q)}$ are coefficients characterizing the medium volumetric changes due to diffusion; $D_{i j}^{*(q)}$ are the self-diffusion coefficients; $R$ is the universal gas constant; $T_{0}$ is the medium temperature; $m^{(q)}$ is the molar mass of $q$-th component; $F_{i}^{*}$ and $Y^{(q)}$ are the mechanical and diffusive bulk perturbations; $g^{(q t)}$ is the Darken thermodynamical factor; $\tau^{(q)}$ is the relaxation time of diffusion perturbations.

To construct equations for the bending of the plate, we turn to the variational formulation of the problem. According to the d'Alembert variational principle, the relations (1) - (3) can be written in the form [10]
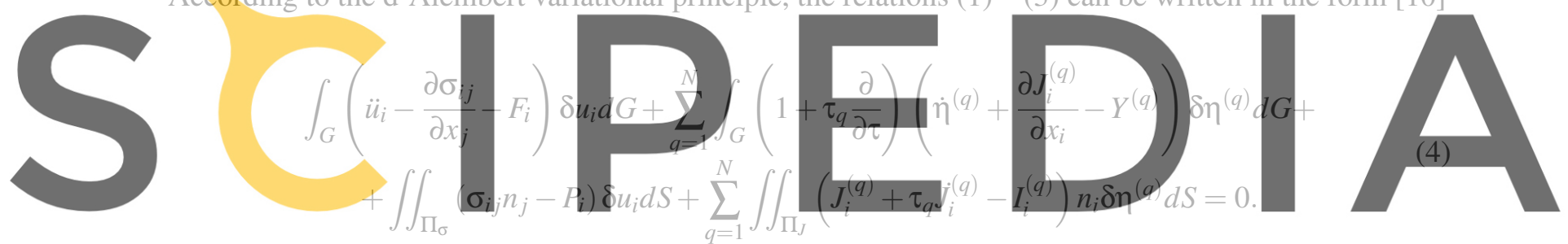

Register for free at https//www.scipedia. com to download the versip n without the watermark

turbances; $G$ is the problem solution domain; $n_{i}$ are components of the outer normal unit vector to $\partial G$, $\partial G=\Pi_{J} \cup \Pi_{\sigma}$.

Further, we assume that:

1. The problem solution domain is rectangular parallelepiped $G=D \times[-h / 2, h / 2]$, where $D=$ $\left[0, l_{1}\right] \times\left[0, l_{2}\right]$ is a rectangular domain in the plate middle surface $x_{3}=0, \Gamma=\partial D$ is boundary domain (fig. 1).

2. Plate surface is $\Pi=\Pi_{-} \cup \Pi_{+} \cup \Pi_{b}$, where $\Pi_{-}$is the bottom surface corresponding to $x_{3}=-h / 2$, $\Pi_{+}$is the top surface corresponding to $x_{3}=h / 2, \Pi_{b}=\Pi_{11} \cup \Pi_{21} \cup \Pi_{12} \cup \Pi_{22}$ is the lateral surface. Here, lateral surfaces $\Pi_{1 k}$ corresponding to $x_{k}=0$, the surfaces $\Pi_{2 k}$ corresponding to $x_{k}=l_{k}$, $k=1,2$. It is assumed that the bottom/top surface is free of mechanical loads

$$
\left.\sigma_{i j} n_{j}\right|_{\Pi_{-}}=\left.\sigma_{i j} n_{j}\right|_{\Pi_{+}}=0
$$

We will also assume that mass transfer through the bottom/top surface is absent 


$$
\left.J_{i}^{(q)}\right|_{\Pi_{-}}=\left.J_{i}^{(q)}\right|_{\Pi_{+}}=0 .
$$

3. The plate material is a homogeneous isotropic continuum

$$
C_{i j k l}=\lambda \delta_{i j} \delta_{k l}+\mu\left(\delta_{i k} \delta_{j l}+\delta_{i l} \delta_{j k}\right), \Lambda_{\alpha \alpha \beta \beta}^{(q)}=\Lambda_{q}, \alpha_{\alpha \alpha}^{(q)}=\alpha_{q}, D_{\alpha \alpha}^{(q)}=D_{q} .
$$

Here $\lambda$ and $\mu$ are Lame coefficients, $\delta_{i j}$ are Kronecker symbol. Due to (3) $\lambda+2 \mu=1$.

4. Transverse deflections are considered small. Then the linearization of the unknown quantities with respect to the variable $x_{3}$ will have the form $[7,8,9,10]$

$$
\begin{gathered}
u_{1}\left(x_{1}, x_{2}, x_{3}, \tau\right)=u\left(x_{1}, x_{2}, \tau\right)-x_{3} \chi_{1}\left(x_{1}, x_{2}, \tau\right), \\
u_{2}\left(x_{1}, x_{2}, \tau\right)=v\left(x_{1}, x_{2}, \tau\right)-x_{3} x_{1}\left(x_{1}, x_{2}, \tau\right), \\
u_{3}\left(x_{1}, x_{2}, \tau\right)=w\left(x_{1}, x_{2}, \tau\right)+x_{3} \psi\left(x_{1}, x_{2}, \tau\right), \\
\eta(q)=N_{q}\left(x_{1}, x_{2}, \tau\right)+x_{3} H_{q}\left(x_{1}, x_{2}, \tau\right) .
\end{gathered}
$$

5. We also consider that a straight fiber normal to the middle surface after deformation also remains straight and normal to the middle surface (the Kirchhoff plate theory). We assume that there are no deformations along the axis $\mathrm{Ox}_{3}$
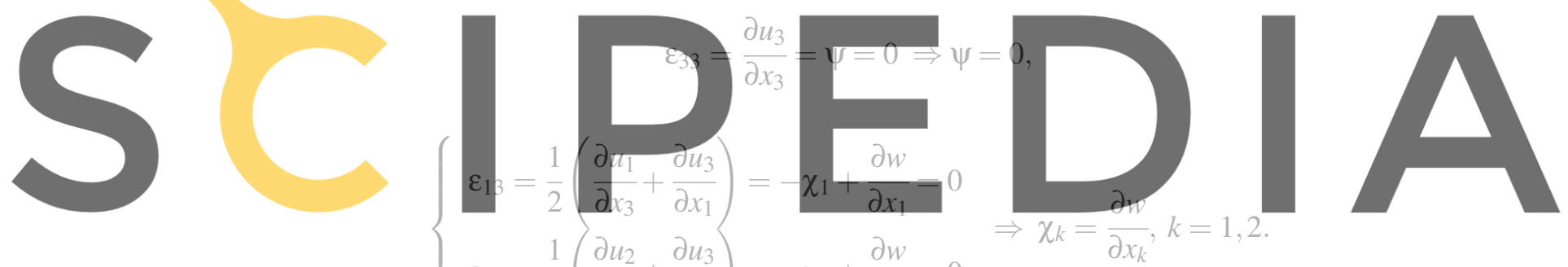

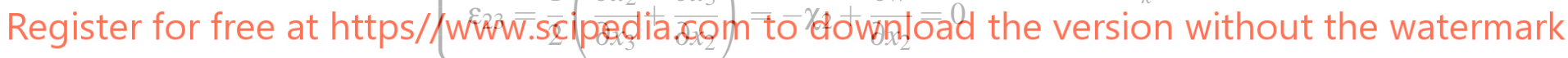

Therefore, the equalities (6) are written as follows:

$$
u_{1}=u-x_{3} \frac{\partial w}{\partial x_{1}}, u_{2}=v-x_{3} \frac{\partial w}{\partial x_{2}}, u_{3}=w, \eta^{(q)}=N_{q}+x_{3} H_{q} .
$$

Then, the components of the stress tensor and the diffusion flux vector will have the form

$$
\begin{gathered}
\sigma_{11}=\frac{\partial u}{\partial x_{1}}-x_{3} \frac{\partial^{2} w}{\partial x_{1}^{2}}+\lambda\left(\frac{\partial v}{\partial x_{2}}-x_{3} \frac{\partial^{2} w}{\partial x_{2}^{2}}\right)-\sum_{q=1}^{N} \alpha_{q}\left(N_{q}+x_{3} H_{q}\right), \\
\sigma_{22}=\lambda\left(\frac{\partial u}{\partial x_{1}}-x_{3} \frac{\partial^{2} w}{\partial x_{1}^{2}}\right)+\frac{\partial v}{\partial x_{2}}-x_{3} \frac{\partial^{2} w}{\partial x_{2}^{2}}-\sum_{q=1}^{N} \alpha_{q}\left(N_{q}+x_{3} H_{q}\right), \\
\sigma_{33}=\lambda\left(\frac{\partial u}{\partial x_{1}}-x_{3} \frac{\partial^{2} w}{\partial x_{1}^{2}}\right)+\lambda\left(\frac{\partial v}{\partial x_{2}}-x_{3} \frac{\partial^{2} w}{\partial x_{2}^{2}}\right)-\sum_{q=1}^{N} \alpha_{q} H_{q},
\end{gathered}
$$




$$
\begin{gathered}
\sigma_{12}=\mu\left(\frac{\partial u}{\partial x_{2}}-2 x_{3} \frac{\partial^{2} w}{\partial x_{1} \partial x_{2}}+\frac{\partial v}{\partial x_{1}}\right), \\
\sigma_{13}=\mu\left(-\frac{\partial w}{\partial x_{1}}+\frac{\partial w}{\partial x_{1}}\right)=0, \sigma_{23}=\mu\left(-\frac{\partial w}{\partial x_{2}}+\frac{\partial w}{\partial x_{2}}\right)=0, \\
J_{1}^{(q)}+\tau_{q} j_{1}^{(q)}=-D_{q}\left(\frac{\partial N_{q}}{\partial x_{1}}+x_{3} \frac{\partial H_{q}}{\partial x_{1}}\right)+\Lambda_{q}\left(\frac{\partial^{2} u}{\partial x_{1}^{2}}-x_{3} \frac{\partial^{3} w}{\partial x_{1}^{3}}\right)+\Lambda_{q}\left(\frac{\partial^{2} v}{\partial x_{1} \partial x_{2}}-x_{3} \frac{\partial^{3} w}{\partial x_{1} \partial x_{2}^{2}}\right), \\
J_{2}^{(q)}+\tau_{q} j_{2}^{(q)}=-D_{q}\left(\frac{\partial N_{q}}{\partial x_{2}}+x_{3} \frac{\partial H_{q}}{\partial x_{2}}\right)+\Lambda_{q}\left(\frac{\partial^{2} u}{\partial x_{1} \partial x_{2}}-x_{3} \frac{\partial^{3} w}{\partial x_{1}^{2} \partial x_{2}}\right)+\Lambda_{q}\left(\frac{\partial^{2} v}{\partial x_{2}^{2}}-x_{3} \frac{\partial^{3} w}{\partial x_{2}^{3}}\right), \\
J_{3}^{(q)}+\tau_{q} \dot{J}_{3}^{(q)}=-D_{q} H_{q}-\Lambda_{q} \frac{\partial^{2} w}{\partial x_{1}^{2}}-\Lambda_{q} \frac{\partial^{2} w}{\partial x_{2}^{2}},(q=\overline{1, N}) .
\end{gathered}
$$

6. A linear transverse load-deflection relationship is presumed (Winkler Model) [14]

$$
q=\widetilde{q}-c_{w} w, c_{w}=\frac{c_{w}^{*} l}{C_{111}^{*}} .
$$

where $c_{w}^{*}$ is the foundation modulus.

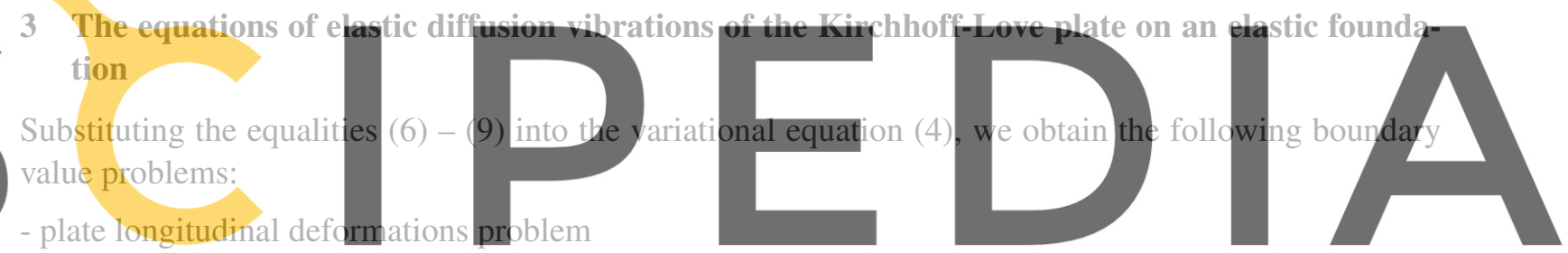

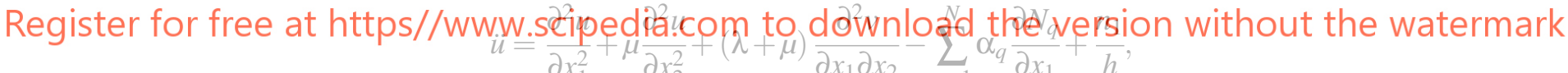

$$
\begin{gathered}
\ddot{v}=(\lambda+\mu) \frac{\partial^{2} u}{\partial x_{1} \partial x_{2}}+\frac{\partial^{2} v}{\partial x_{2}^{2}}+\mu \frac{\partial^{2} v}{\partial x_{1}^{2}}-\sum_{q=1}^{N} \alpha_{q} \frac{\partial N_{q}}{\partial x_{2}}-\frac{n_{2}}{h} \\
\dot{N}_{q}+\tau_{q} \ddot{N}_{q}=D_{q}\left(\frac{\partial^{2} N_{q}}{\partial x_{1}^{2}}+\frac{\partial^{2} N_{q}}{\partial x_{2}^{2}}\right)-\Lambda_{q}\left(\frac{\partial^{3} u}{\partial x_{1}^{3}}+\frac{\partial^{3} v}{\partial x_{1}^{2} \partial x_{2}}+\frac{\partial^{3} u}{\partial x_{1} \partial x_{2}^{2}}+\frac{\partial^{3} v}{\partial x_{2}^{3}}\right)+\frac{y_{q}}{h} ;
\end{gathered}
$$

- plate deflections problem

$$
\begin{gathered}
\frac{\partial^{2} \ddot{w}}{\partial x_{1}^{2}}+\frac{\partial^{2} \ddot{w}}{\partial x_{2}^{2}}-\frac{12}{h^{2}} \ddot{w}=\frac{\partial^{4} w}{\partial x_{2}^{4}}+\frac{\partial^{4} w}{\partial x_{1}^{4}}+2 \frac{\partial^{4} w}{\partial x_{1}^{2} \partial x_{2}^{2}}+\frac{12}{h^{3}} c_{w} w+ \\
+\sum_{q=1}^{N} \alpha_{q}\left(\frac{\partial^{2} H_{q}}{\partial x_{2}^{2}}+\frac{\partial^{2} H_{q}}{\partial x_{1}^{2}}\right)-\frac{12}{h^{3}}\left(\frac{\partial m_{2}}{\partial x_{2}}+\frac{\partial m_{1}}{\partial x_{1}}+q\right), \\
\dot{H}_{q}+\tau_{q} \ddot{H}_{q}=D_{q}\left(\frac{\partial^{2} H_{q}}{\partial x_{1}^{2}}+\frac{\partial^{2} H_{q}}{\partial x_{2}^{2}}\right)+\Lambda_{q}\left(\frac{\partial^{4} w}{\partial x_{1}^{4}}+2 \frac{\partial^{4} w}{\partial x_{1}^{2} \partial x_{2}^{2}}+\frac{\partial^{4} w}{\partial x_{2}^{4}}\right)+\frac{12}{h^{3}} z_{q} .
\end{gathered}
$$


The unsteady bending mathematical model of a simply supported plate on an elastic foundation under a distributed load action is described by the equations (10), which are supplemented by zero initial conditions and boundary conditions, which are also obtained from the variational equation (4)

$$
\begin{gathered}
\left.\left(\frac{\partial^{2} w}{\partial x_{1}^{2}}+\lambda \frac{\partial^{2} w}{\partial x_{2}^{2}}+\sum_{q=1}^{N} \alpha_{q} H_{q}\right)\right|_{x_{1}=0}=0,\left.\left(\frac{\partial^{2} w}{\partial x_{1}^{2}}+\lambda \frac{\partial^{2} w}{\partial x_{2}^{2}}+\sum_{q=1}^{N} \alpha_{q} H_{q}\right)\right|_{x_{1}=l_{1}}=0 \\
\left.\left(\lambda \frac{\partial^{2} w}{\partial x_{1}^{2}}+\frac{\partial^{2} w}{\partial x_{2}^{2}}+\sum_{q=1}^{N} \alpha_{q} H_{q}\right)\right|_{x_{2}=0}=0,\left.\left(\lambda \frac{\partial^{2} w}{\partial x_{1}^{2}}+\frac{\partial^{2} w}{\partial x_{2}^{2}}+\sum_{q=1}^{N} \alpha_{q} H_{q}\right)\right|_{x_{2}=l_{2}}=0 \\
\left.w\right|_{x_{1}=0}=0,\left.w\right|_{x_{1}=l_{1}}=0,\left.w\right|_{x_{2}=0}=0,\left.w\right|_{x_{2}=l_{2}}=0 ; \\
\left.H_{q}\right|_{x_{1}=0}=0,\left.H_{q}\right|_{x_{1}=l_{1}}=0,\left.H_{q}\right|_{x_{2}=0}=0,\left.H_{q}\right|_{x_{2}=l_{2}}=0 .
\end{gathered}
$$

\section{Solution method}

The solutions of the problem (10) - (13) under the action of distributed disturbances $F_{1}\left(x_{1}, x_{2}, \tau\right)=$ $-12(\widetilde{q}+\operatorname{divm}) / h^{3}$ and $F_{q+1}\left(x_{1}, x_{2}, \tau\right)=12 z^{(q)} / h^{3}$ are sought in integral form $(q=\overline{1, N+1})$ :
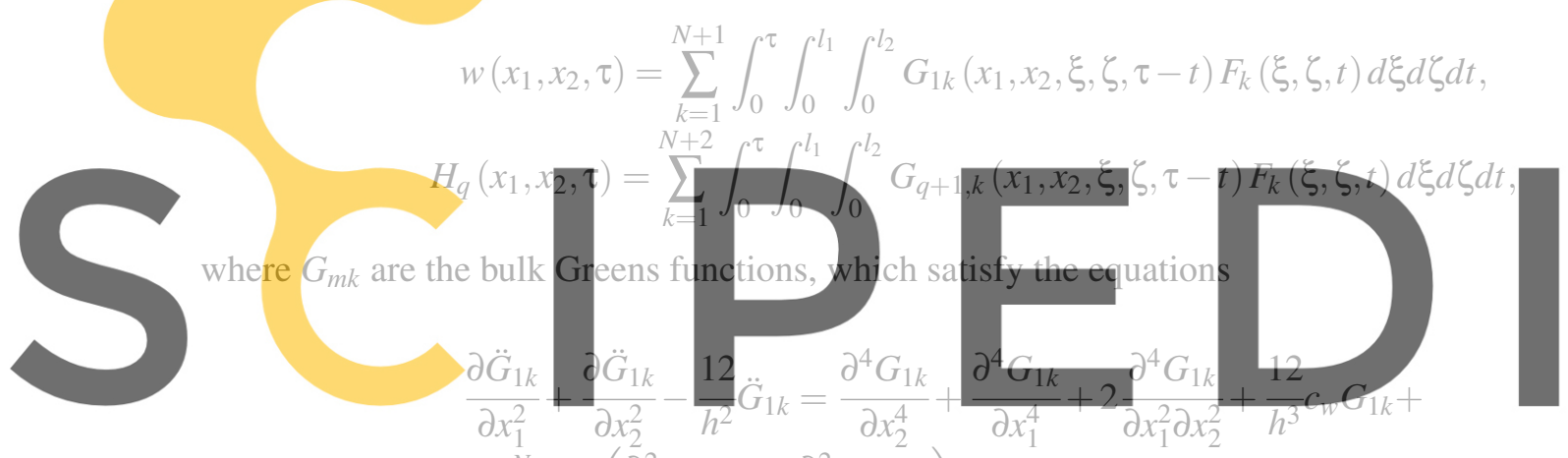

Register for free at https\$ywoww.scipedia.com to dowbrtoad the version, without the watermark

$$
\begin{gathered}
\dot{G}_{q+1, k}+\tau_{q} \ddot{G}_{q+1, k}=D_{q}\left(\frac{\partial^{2} G_{q+1, k}}{\partial x_{1}^{2}}+\frac{\partial^{2} G_{q+1, k}}{\partial x_{2}^{2}}\right)+\Lambda_{q}\left(\frac{\partial^{4} G_{1 k}}{\partial x_{1}^{4}}+2 \frac{\partial^{4} G_{1 k}}{\partial x_{1}^{2} \partial x_{2}^{2}}+\frac{\partial^{4} G_{1 k}}{\partial x_{2}^{4}}\right)+ \\
+\delta_{q+1, k} \boldsymbol{\delta}\left(x_{1}-\xi\right) \delta\left(x_{2}-\zeta\right) \boldsymbol{\delta}(\tau)
\end{gathered}
$$

and homogeneous boundary conditions

$$
\begin{gathered}
\left.\left(\frac{\partial^{2} G_{1 k}}{\partial x_{1}^{2}}+\lambda \frac{\partial^{2} G_{1 k}}{\partial x_{2}^{2}}+\sum_{q=1}^{N} \alpha_{q} G_{q+1, k}\right)\right|_{x_{1}=0}=0,\left.\left(\frac{\partial^{2} G_{1 k}}{\partial x_{1}^{2}}+\lambda \frac{\partial^{2} G_{1 k}}{\partial x_{2}^{2}}+\sum_{q=1}^{N} \alpha_{q} G_{q+1, k}\right)\right|_{x_{1}=l_{1}}=0, \\
\left.\left(\lambda \frac{\partial^{2} G_{1 k}}{\partial x_{1}^{2}}+\frac{\partial^{2} G_{1 k}}{\partial x_{2}^{2}}+\sum_{q=1}^{N} \alpha_{q} G_{q+1, k}\right)\right|_{x_{2}=0}=0,\left.\left(\lambda \frac{\partial^{2} G_{1 k}}{\partial x_{1}^{2}}+\frac{\partial^{2} G_{1 k}}{\partial x_{2}^{2}}+\sum_{q=1}^{N} \alpha_{q} G_{q+1, k}\right)\right|_{x_{2}=l_{2}}=0, \\
\left.G_{1 k}\right|_{x_{1}=0}=0,\left.G_{1 k}\right|_{x_{1}=l_{1}}=0,\left.G_{1 k}\right|_{x_{2}=0}=0,\left.G_{1 k}\right|_{x_{2}=l_{2}}=0,
\end{gathered}
$$




$$
\left.G_{q+1, k}\right|_{x_{1}=0}=0,\left.G_{q+1, k}\right|_{x_{1}=l_{1}}=0,\left.G_{q+1, k}\right|_{x_{2}=0}=0,\left.G_{q+1, k}\right|_{x_{2}=l_{2}}=0 .
$$

To find the Green's functions $G_{i k}$ the expanding into double trigonometric Fourier series in spatial coordinate and the Laplace transform in time are use. As a result, problem (15), (16) is reduced to the following system of linear algebraic equations ( $s$ is Laplace transform parameter, $\lambda_{n}=\pi n / l_{1}, \mu_{m}=\pi m / l_{2}$ )

$$
\begin{gathered}
k_{1}\left(v_{n m}, s\right) G_{1 k}^{L s s}\left(\lambda_{n}, \mu_{m}, \xi, \zeta, s\right)-\sum_{q=1}^{N} \alpha_{q} v_{n m}^{2} G_{q+1, k}^{L s s}\left(\lambda_{n}, \mu_{m}, \xi, \zeta, s\right)=\frac{4}{l_{1} l_{2}} \delta_{1 k} \sin \mu_{m} \zeta \sin \lambda_{n} \xi, \\
-\Lambda_{q} v_{n m}^{4} G_{1 k}^{L s s}\left(\lambda_{n}, \mu_{m}, \xi, \zeta, s\right)+k_{q+1}\left(v_{n m}, s\right) G_{q+1, k}^{L s s}\left(\lambda_{n}, \mu_{m}, \xi, \zeta, s\right)=\frac{4}{l_{1} l_{2}} \delta_{q+1, k} \sin \mu_{m} \zeta \sin \lambda_{n} \xi .
\end{gathered}
$$

Here

$$
\begin{gathered}
k_{1}\left(v_{n m}, s\right)=s^{2}\left(v_{n m}^{2}+\frac{12}{h^{2}}\right)+v_{n m}^{4}+\frac{12}{h^{3}} c_{w}, k_{q+1}\left(v_{n m}, s\right)=s+\tau_{q} s^{2}+D_{q} v_{n m}^{2}, v_{n m}^{2}=\lambda_{n}^{2}+\mu_{m}^{2}, \\
G_{i k}^{L s s}\left(\lambda_{n}, \mu_{m}, \xi, \zeta, s\right)=\frac{4}{l_{1} l_{2}} \int_{0}^{l_{1}} \int_{0}^{l_{2}} G_{i k}^{L}\left(x_{1}, x_{2}, \xi, \zeta, s\right) \sin \lambda_{n} x_{1} \sin \mu_{m} x_{2} d x_{2} d x_{1},
\end{gathered}
$$
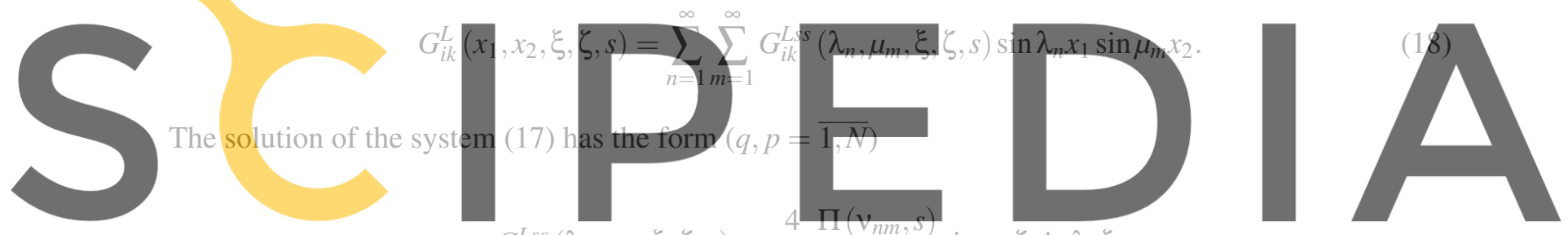

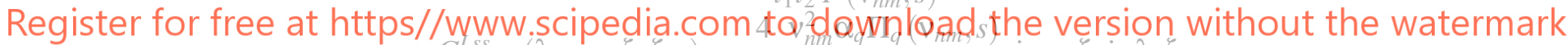
$G_{1, q+1}^{L s s}\left(\lambda_{n}, \mu_{m}, \xi, \zeta, s\right)=\frac{L_{1}}{l_{1} l_{2}} \frac{m_{m}}{P\left(v_{n m}, s\right)} \sin \mu_{m} \xi \sin \lambda_{n} \xi$,

$$
\begin{gathered}
G_{q+1,1}^{L s s}\left(\lambda_{n}, \mu_{m}, \xi, \zeta, s\right)=\frac{4}{l_{1} l_{2}} \frac{\Lambda_{q} v_{n m}^{4} \Pi\left(v_{n m}, s\right)}{Q_{q}\left(v_{n m}, s\right)} \sin \mu_{m} \zeta \sin \lambda_{n} \xi \\
G_{q+1, p+1}^{L s s}\left(\lambda_{n}, \mu_{m}, \xi, \zeta, s\right)=\frac{4}{l_{1} l_{2}}\left[\frac{\delta_{q p}}{k_{q+1}\left(v_{n m}, s\right)}+\frac{\Lambda_{q} v_{n m}^{6} \alpha_{p} \Pi_{p}\left(v_{n m}, s\right)}{Q_{q}\left(v_{n m}, s\right)}\right] \sin \mu_{m} \zeta \sin \lambda_{n} \xi
\end{gathered}
$$

where

$$
\begin{gathered}
\Pi\left(v_{n m}, s\right)=\prod_{j=1}^{N} k_{j+1}\left(v_{n m}, s\right), \Pi_{j}\left(v_{n m}, s\right)=\prod_{r=1, r \neq j}^{N} k_{r+1}\left(v_{n m}, s\right), \\
P\left(v_{n m}, s\right)= \\
k_{1}\left(v_{n m}, s\right) \Pi\left(v_{n m}, s\right)-v_{n m}^{6} \sum_{j=1}^{N} \alpha_{j} \Lambda_{j} \Pi_{j}\left(v_{n m}, s\right), \\
Q_{q}\left(v_{n m}, s\right)=k_{q}\left(v_{n m}, s\right) P\left(v_{n m}, s\right) .
\end{gathered}
$$


The originals in equalities (19) are calculated on the base of residues and the tables of operational calculus (prime denotes derivative with respect to parameter $s$ ) [15]

$$
\begin{gathered}
G_{1 k}^{s s}\left(\lambda_{n}, \mu_{m}, \xi, \zeta, \tau\right)=\frac{4}{l_{1} l_{2}} \sin \mu_{m} \zeta \sin \lambda_{n} \xi \sum_{l=1}^{2 N+2} A_{1 k}^{(l)}\left(v_{n m}, s_{l}\left(v_{n m}\right)\right) \exp \left(s_{l}\left(v_{n m}\right) \tau\right), \\
G_{1, q+1}^{s s}\left(\lambda_{n}, \mu_{m}, \xi, \zeta, \tau\right)=\frac{4}{l_{1} l_{2}} \sin \mu_{m} \zeta \sin \lambda_{n} \xi \sum_{l=1}^{2 N+2} A_{q+1, k}^{(l)}\left(v_{n m}, s_{l}\left(v_{n m}\right)\right) \exp \left(s_{l}\left(v_{n m}\right) \tau\right), \\
G_{q+1,1}^{s s}\left(\lambda_{n}, \mu_{m}, \xi, \zeta, \tau\right)=\frac{4}{l_{1} l_{2}} \sin \mu_{m} \zeta \sin \lambda_{n} \xi \sum_{l=1}^{2 N+4} A_{q+1,1}^{(l)}\left(v_{n m}, s_{l}\left(v_{n m}\right)\right) \exp \left(s_{l}\left(v_{n m}\right) \tau\right), \\
G_{q+1, p+1}^{s s}\left(\lambda_{n}, \mu_{m}, \xi, \zeta, \tau\right)=\frac{4}{l_{1} l_{2}} \sum_{l=1}^{2} \frac{\exp \left(\chi_{l}\left(v_{n m}\right) \tau\right)}{k_{q+1}^{\prime}\left(v_{n m}, s\right)} \sin \mu_{m} \zeta \sin \lambda_{n} \xi+ \\
+\frac{4}{l_{1} l_{2}} \sin \mu_{m} \zeta \sin \lambda_{n} \xi \sum_{l=1}^{2 N+4} A_{q+1, p+1}^{(l)}\left(v_{n m}, s_{l}\left(v_{n m}\right)\right) \exp \left(s_{l}\left(v_{n m}\right) \tau\right),
\end{gathered}
$$

where

$$
\begin{gathered}
A_{1 k}^{(l)}\left(\mathrm{v}_{n m}, s_{l}\left(\mathrm{v}_{n m}\right)\right)=\frac{\Pi\left(\mathrm{v}_{n m}, s_{l}\left(\mathrm{v}_{n m}\right)\right)}{P^{\prime}\left(\mathrm{v}_{n m}, s_{l}\left(\mathrm{v}_{n m}\right)\right)}, \\
A_{q+1, k}^{(l)}\left(\mathrm{v}_{n m}, s_{l}\left(\mathrm{v}_{n m}\right)\right)=\frac{\mathrm{v}_{n m}^{2} \alpha_{q} \Pi_{q}\left(\mathrm{v}_{n m}, s_{l}\left(\mathrm{v}_{n m}\right)\right)}{P^{\prime}\left(\mathrm{v}_{n m}, s_{l}\left(\mathrm{v}_{n m}\right)\right)},
\end{gathered}
$$
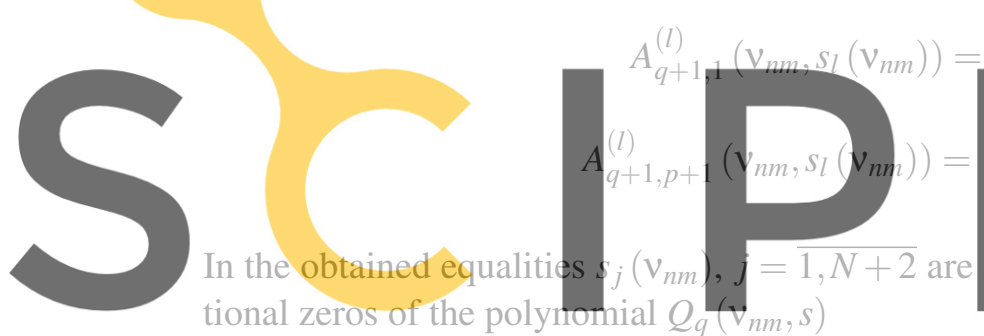

$$
\Lambda_{q} \mathrm{v}_{n m}^{4} \Pi\left(\mathrm{v}_{n m}, s_{l}\left(\mathrm{v}_{n m}\right)\right)
$$
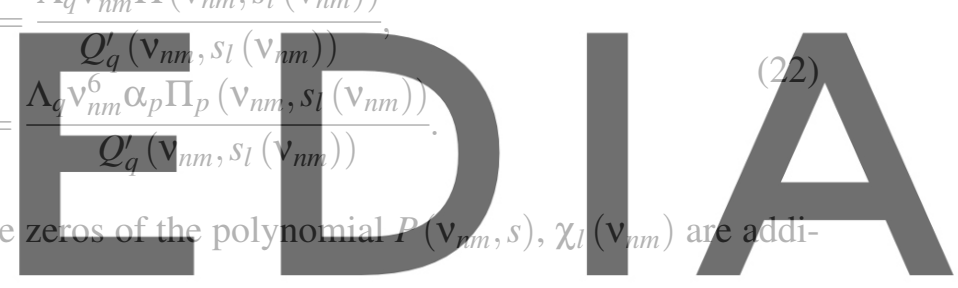

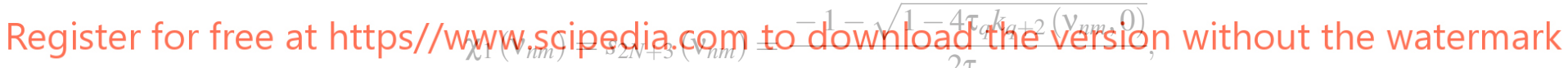

$$
\chi_{2}\left(\mathbf{v}_{n m}\right)=s_{2 N+4}\left(\mathbf{v}_{n m}\right)=\frac{-1+\sqrt{1-4 \tau_{q} k_{q+2}\left(v_{n m}, 0\right)}}{2 \tau_{q}} .
$$

\section{Example}

Take calculating the material with the following characteristics (duralumin, $\lambda^{*}$ and $\mu^{*}$ are Lame coefficients) [16]:

$$
\begin{gathered}
\lambda^{*}=6.93 \cdot 10^{10} \frac{N}{\mathrm{~m}^{2}}, \mu^{*}=2.56 \cdot 10^{10} \frac{\mathrm{N}}{\mathrm{m}^{2}}, T_{0}=800 \mathrm{~K}, \rho=2700 \frac{\mathrm{kg}}{\mathrm{m}^{3}}, \mathrm{~h}=10^{-3} \mathrm{~m} \\
\alpha_{11}^{*(1)}=\alpha_{22}^{*(1)}=1.55 \cdot 10^{7} \frac{\mathrm{J}}{\mathrm{kg}}, \alpha_{11}^{*(2)}=\alpha_{22}^{*(2)}=6.14 \cdot 10^{7} \frac{\mathrm{J}}{\mathrm{kg}} \\
D_{11}^{*(1)}=D_{22}^{*(1)}=7.73 \cdot 10^{-14} \frac{\mathrm{m}^{2}}{\mathrm{~s}}, D_{11}^{*(2)}=D_{22}^{*(2)}=6.67 \cdot 10^{-14} \frac{\mathrm{m}^{2}}{\mathrm{~s}} \\
n_{0}^{(1)}=0.95, n_{0}^{(2)}=0.05, \mathrm{~m}^{(1)}=0.027 \frac{\mathrm{kg}}{\mathrm{mol}}, \mathrm{m}^{(2)}=0.064 \frac{\mathrm{kg}}{\mathrm{mol}} .
\end{gathered}
$$



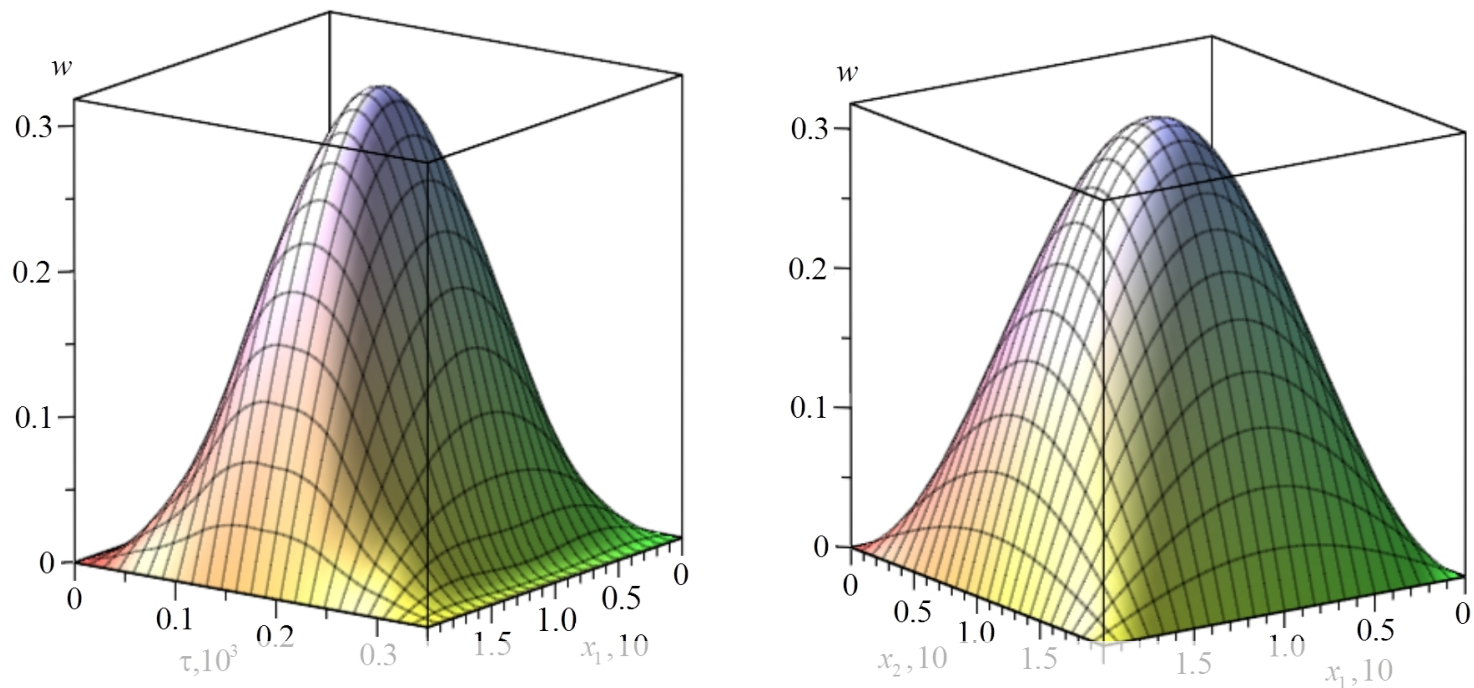

Figure 2: The plate deflections $w\left(x_{1}, l_{2} / 2, \tau\right), c_{w}^{*} l=$ $1.25 \cdot 10^{7} \mathrm{~N} / \mathrm{m}^{2}$

We assume that the plate is rectangular $l_{1}^{*}=0.01 \mathrm{~m}, l_{2}^{*}=0.01 \mathrm{~m}$ : thickness $h^{*}=0.001 \mathrm{~m}$. We set the load in the form
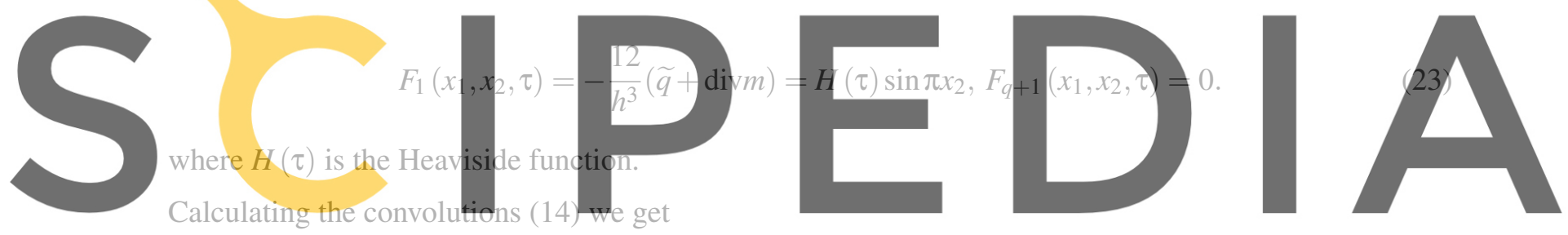

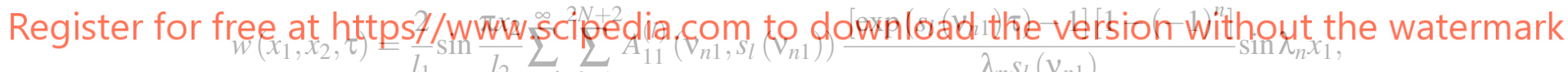

$$
H_{q}\left(x_{1}, x_{2}, \tau\right)=\frac{2}{l_{1}} \sin \frac{\pi x_{2}}{l_{2}} \sum_{n=1}^{\infty} \sum_{l=1}^{2 N+4} A_{q+1,1}^{(l)}\left(v_{n 1}, s_{l}\left(v_{n 1}\right)\right) \frac{\left[\exp \left(s_{l}\left(v_{n 1}\right) \tau\right)-1\right]\left[1-(-1)^{n}\right]}{\lambda_{n} s_{l}\left(v_{n 1}\right)} \sin \lambda_{n} x_{1}
$$

The calculation results are presented in Figures $2-7$. Figure 2 shows the plate deflections on an elastic foundation. Figure 3 shows deflections in the absence of an elastic foundation.

Based on the performed calculations, it can be seen that the unsteady plate bending initiates diffusion flows in the plate. The Figure 4 shows the linear density of the aluminum concentration increment during elastic diffusion vibrations of the plate on an elastic foundation. In the Figure 5 is the same, but in the absence of an elastic foundation.

In Figures 6-9 are demonstrate the diffusion field effect on the displacement field.

The Figures 7 and 9 show the case when there is no elastic foundation. It is shown that, starting from a certain time, the elastic-diffusion vibrations begin to lag behind the elastic vibrations. In the presence of an elastic foundation, the influence of diffusion effects becomes negligible. The Figures 6,8 show the 

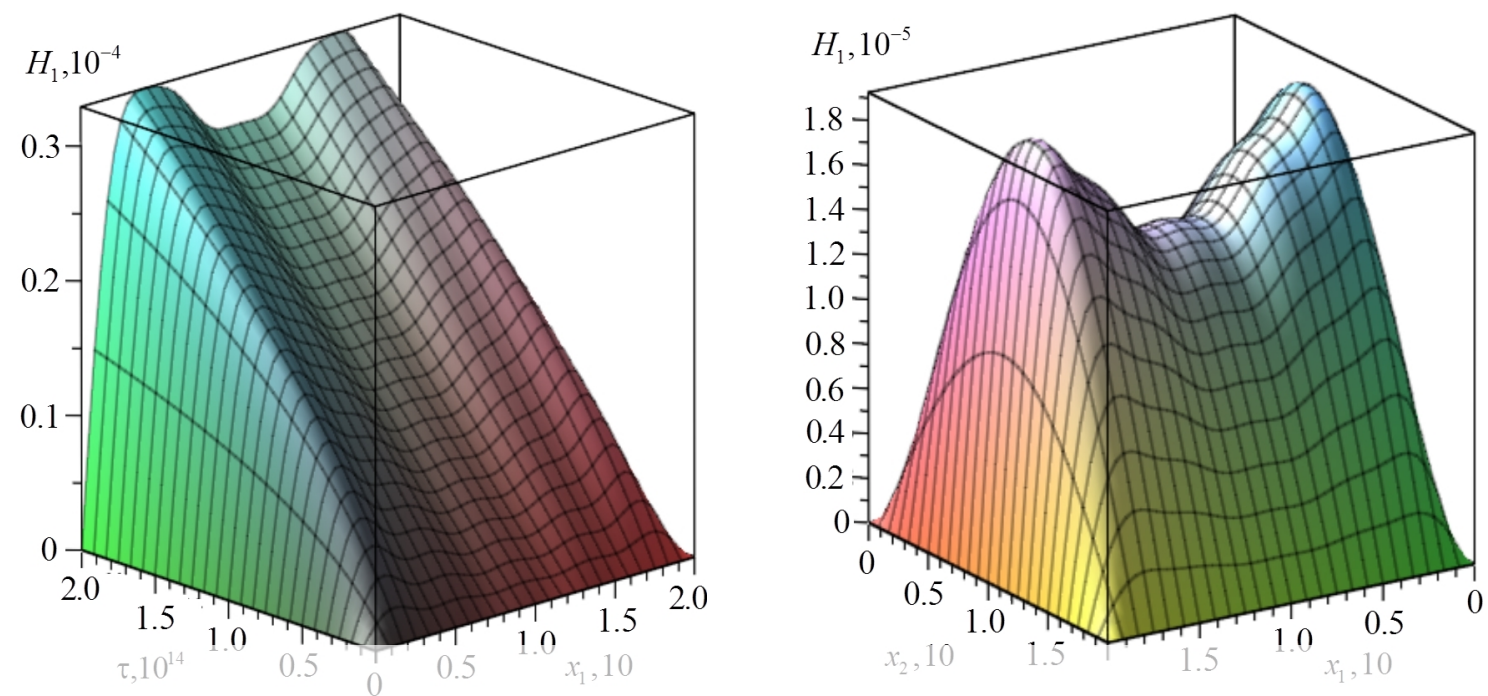

Figure 4: The concentration increment $H_{1}\left(x_{1}, l_{2} / 2, \tau\right)$, Figure 5: The concentration increment $H_{1}\left(x_{1}, l_{2} / 2, \tau\right)$, $c_{w}^{*} l=1.25 \cdot 10^{7} \mathrm{~N} / \mathrm{m}^{2}$ $c_{w}^{*}=0$

comparative results of elastic and elastic diffusion vibrations for the plate on an elastic foundation $c_{w}^{*} l=$ $1.25 \cdot 10^{7} \mathrm{~N} / \mathrm{m}^{2}$. At a sufficiently long time interval $\tau \sim 10^{12}$, the effect of diffusion on the displacement field was not found.

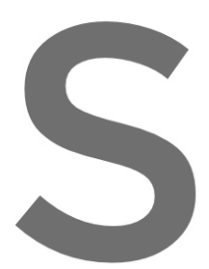

6 CONCLUSIONS

The unsteady vibrations $\mathrm{m}$ foundation is constructed. diffusion fields in continutum. $A$ i
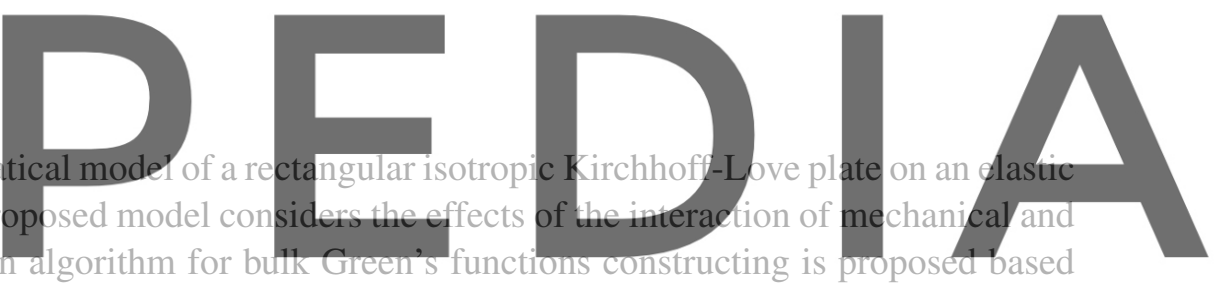

on the use of the Laplace transform and expansions in trigonometric Fourier series.

Register for free at https//www.scipedia.com to download the version without the watermark By the example of plate bending under the action of a distributed mechanical load, the effect of interaction between mechanical and diffusion fields is demonstrated. It is shown that, on the one hand, unsteady bending initiates the process of mass transfer. On the other hand, diffusion effects the displacement field, which manifests itself in the form of a delay in mechanodiffusion oscillations with respect to purely mechanical ones. However, the presence of an elastic foundation significantly reduces the effect of diffusion on the displacement field. These results are presented in analytical and graphical forms.

\section{REFERENCES}

[1] Shvets R.N., Flyachok V.M.: Uravneniya mexanodiffuzii anizotropny 'ch obolochek s uchetom poperechny 'ch deformacij [The equations of mechanothermodiffusion of anisotropic shells taking account of transverse strains]. Mat. Met. Fiz.-Mekh. Polya (1984) 20: 54-61. [In Russian].

[2] Shvets R.N., Flyachok V.M. Variacionny‘j podxod k resheniyu dinamicheskich zadach mexanotermodiffuzii anizotropny'ch obolochek [A variational approach to solving dynamic problems of mechanothermal diffusion of anisotropic shells]. Mat. fiz. i nelinejnaya mexanika. (1991) 16: 39-43. [In Russian]. 


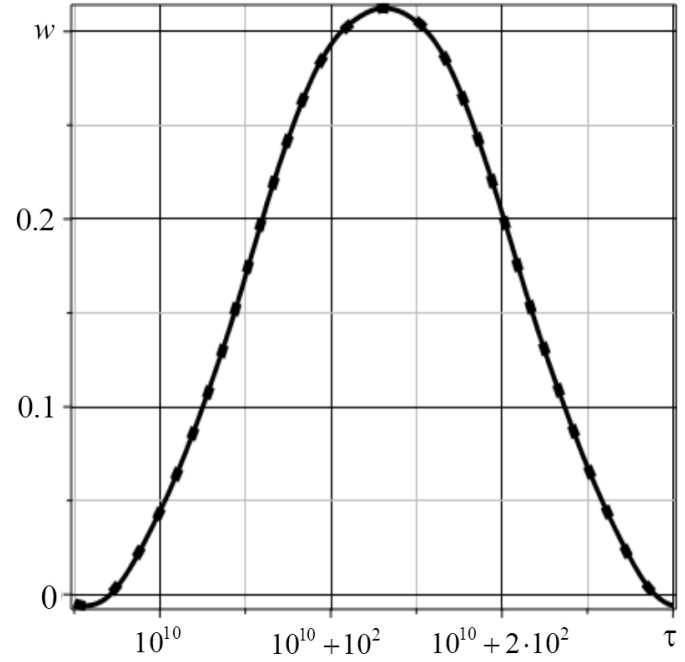

Figure 6: The plate deflections $w\left(\frac{l_{1}}{2}, \frac{l_{2}}{2}, \tau\right), c_{w}^{*} l=1.25$. $10^{7} \mathrm{~N} / \mathrm{m}^{2}$

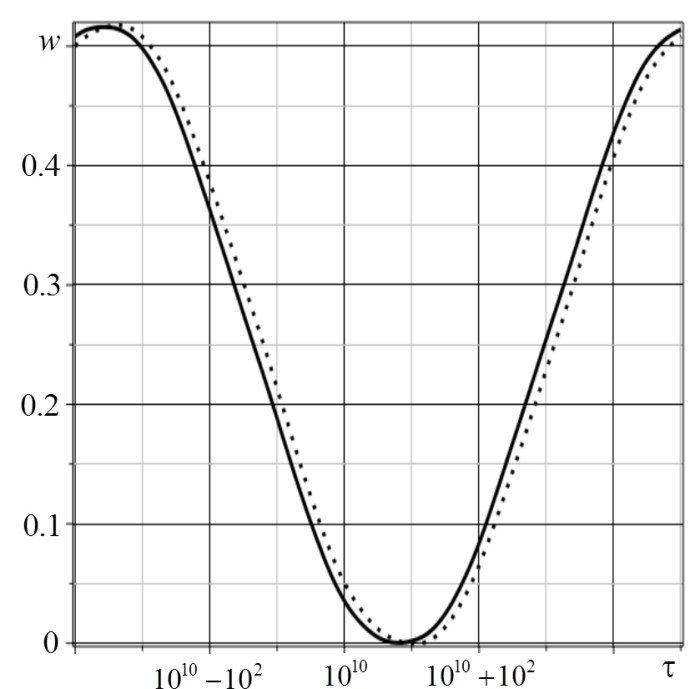

Figure 7: The plate deflections $w\left(\frac{l_{1}}{2}, \frac{l_{2}}{2}, \tau\right), c_{w}^{*}=0$

[3] Aouadi M. Miranville A. Smooth attractor for a nonlinear thermoelastic diffusion thin plate based on Gurtin-Pipkin's model. Asymptotic Analysis. (2015) 95: 129-160.

[4] Aouadi M. On thermoelastic diffusion thin plate theory. Appl. Math. Mech. Engl. Ed. (2015) 36(5): 619-632.

[5] Aouadi M., Miranville A. Quasi-stability and global attractor in nonlinear thermoelastic diffusion plate with memory. Evolution equations and control theory. (2015) 4(3): 241-263.

[6] Bhattacharya D., Kanoria M. The influence of two temperature generalized thermoelastic diffusion inside a spherical shell. International Journal of Engineering and Technical Research (IJETR). (2014) 2(5): 151-159.

[7] Tarlakovskii D.V., Zemskov A.V. An Elastodiffusive Orthotropic Euler-Bernoulli Beam with Considering Diffusion Flux Relaxation. Math. Comput. Appl. (2019) 24(1)23. doi:10.3390/mca24010023.

[8] Afanasieva O.A., Zemskov A.V. Unsteady Elastic-Diffusion Oscillations of a Simply Supported Kirchhoff Plate Under the Distributed Transverse Load Action. In: Gdoutos E., Konsta-Gdoutos M. (eds) Proceedings of the Third International Conference on Theoretical, Applied and Experimental Mechanics. ICTAEM 2020. Structural Integrity, vol 16. Springer, Cham (2020): 181-186.

[9] Le K.C. Vibrations of shells and rods. Berlin, Springer Verlag, (1999), 425 P.

[10] Mikhailova E.Yu., Tarlakovskii D.V., Fedotenkov G.V. Obshchaya teoriya uprugikh obolochek [General theory of anisotropic shells]. Moscow, MAI, (2018) [In Russian].

[11] Igumnov L.À., Tarlakovskii D.V., Zemskov A.V. A two-dimensional nonstationary problem of elastic diffusion for an orthotropic one-component layer. Lobachevskii Journal of Mathematics. (2017) 38(5): 808-817. 


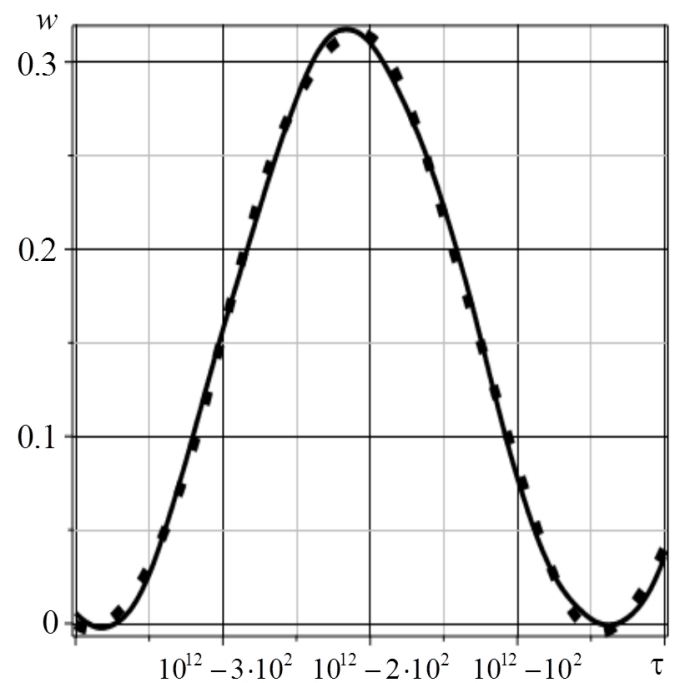

Figure 8: The plate deflections $w\left(\frac{l_{1}}{2}, \frac{l_{2}}{2}, \tau\right), c_{w}^{*} l=1.25$. $10^{7} \mathrm{~N} / \mathrm{m}^{2}$

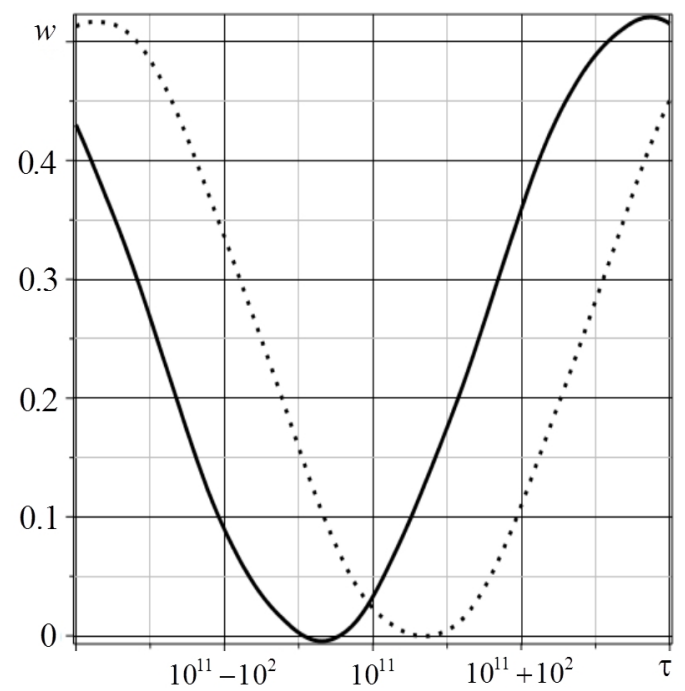

Figure 9: The plate deflections $w\left(\frac{l_{1}}{2}, \frac{l_{2}}{2}, \tau\right), c_{w}^{*}=0$

[12] Zemskov A.V., Tarlakovskiy D.V. Two-dimensional nonstationary problem elastic for diffusion an isotropic one-component layer. Journal of Applied Mechanics and Technical Physics. (2015) 56(6): $1023-1030$.

[13] Tarlakovskii D.V., Vestyak V.A., Zemskov A.V. Dynamic Processes in Thermoelectromagnetoelastic and Thermoelastodiffusive Media. Encyclopedia of thermal stress, volume 2, Springer Dordrecht Heidelberg New York London, Springer reference. (2014): 1064 - 1071.

[14] Shahin, N.A., Mbakisya O. Simply supported beam response on elastic foundation carrying repeated rolling concentrated load. Journal of Engineering Science and Technology. (2010) 5(1): 52-66.

[15] Ditkin V.A., Prudnikov A.P. Spravochnik po operacionnomu ischisleniyu [Operational Calculus Handbook]. Moscow, Vy‘sshaya shkola, (1965) [In Russian].

[16] Fizicheskie velichiny: Spravochnik [Physical quantities: Handbook] / Babichev A.P., Babushkina N.A., Bratkovskij A.M. ets. Edited by Grigor'ev I.S., Melixov I.Z. Moscow, Energoatomizdat, (1991) [In Russian]. 